ISSN No. 0974-035X

An indexed refereed \& peer-reviewed journal of higher education

Towards Excellence

UGC-HUMAN RESOURCE DEVELOPMENT CENTRE

Gujarat University, Ahmedabad-380009, Gujarat, India

\title{
લોકસભાની વર્ષ-૨૦૧૯ની ચંંટણી - એક અભ્યાસ
}

\section{Mr. Parmar Sunil G \\ Dr. Hitesh R. Patel}

\begin{abstract}
ભારતનું મજબૂત લોકતંત્ર વર્ષ-૨૦૧૯મા જે સામાન્ય ચૂંટણી થઈ એમાં જે બહુમતી મેળવી અને આ લોકત્રાંતિક દેશમાં જે લોકોએ વોટ નો અધિકારને લોકપર્વમા ફેરવી જનાદેશનું ઉત્તમ ઉદાહરણ પૂરું પાડ્યું છે આ બહમતીથી ચૂંટાયેલી સરકાર જેમના દૂરદશ્શી દસ્તાવેજનું અમલીકરણ કરી લોકમાનસમાં ઉંડી છાપ છોડશે તેવી આશા રાખવામા આવે છે તેમજ સહયોગી દળોનો જે રીત સાથ અને સહકાર મળી રહ્યો છે અને તેના સૂત્ર પ્રમાણે સૌનો સાથ સૌનો વિકાસ સૌનો વિશ્વાસ સાચા અર્થ માં સાર્થક નીવડે તેવી અપેક્ષા સેવવામાં આવે છે
\end{abstract}

Keywords: લોકતંત્ર, પ્રજાસત્તાક, સહયોગી દળો, દૂરદર્શી દસ્તાવેજી, વોટ, લોકપર્વ, લોકમાનસ, જનાદેશ

\section{પ્રસ્તાવના}

આધુનિક વિશાળ વસ્તીવાળા લોકશાહી દેશોમાં પ્પત્યેક નાગરીક સમાન ગણાય છે. પુખ્ત ઉંમરે તમામે નાગરીકોને મતાધિકાર અપાય છે. નાગરીકો નિશ્ચિત સમયે યોજાતી ચૂંટણીઓમાં ગુપ્ત મતદાનથી પોતાના પ્રતિનિધિઓને ચૂંટતા હોય છે. તથા પ્રતિનિધિઓ દ્વારા પરોક્ષ રીતે લોકશાહી ચાલે છે. તેમજ લોકશાહીમાં પ્રતિનિધિઓની પસંદગી માટેની અગત્યની પ્રુ્રિયા એ ચૂંટણી છે. આમ, લોકશાહી પ્રકારની શાસન વ્યવસ્થા માં ચૂંટણી હાર્દ સમાન ગણાય છે.

સ્વતંત્ર ભારતનું બંધારણ ૨૬ મી જાન્યુઆરી ૧૯૫૦ માં અમલમાં આવ્યું. બંધારણના ૧૫માં ભાગમાં કલમ ૩ર૪ થી ૩ર૯ વચ્ચે ચૂંટણીપંચ તથા ચૂંટણી પ્રક્રિયા માટેની જોગવાઇ છે. આમ, ચૂંટણીપંચ એ બંધારણીય સંસ્થા છે. તે ઉપરાંત સ્વતંત્ર, તટસ્થ અને ન્યાયી ચૂંટણીઓના આયોજન માટે ચૂંટણીપંચે ૧૯૫ર થી ૨૦૧૯ સુધીની ૧૭ લોકસભાની ચૂંટણીઓમાં મહત્ત્વની કામગીરી કરી છે.

ભારતનું બંધારણ અમલમાં આવ્યું ત્યારથી, પ્રથમ સામાન્ય ચૂંટણીઓ માટેની વિચારણા શરૂ થઇ ચૂકી હતી. દેશનું વિભાજન થયેલું હતું. નિર્વાસિતોનો પ્રક્ષ હતો. દેશમાં નિરક્ષરતા અને ગરીબાઇનું મોટું પ્રમાણ હતું. આમ છતાં પ્રજા ચૂંટણીઓ માટે ઉત્સાહ અનુભવતી હતી. 


\section{Parmar \& Dr. Hitesh Patel / Page 130-136}

ભારતમાં ઓક્ટબબર ૧૯૫૧ થી ફેબ્રુઆરી ૧૯૫ર ના સમયગાળા દરમિયાન લોકસભાની પ્રથમ સામાન્ય ચૂંટણી થઇ હતી. જેમાં ૪૮૯ બેઠકો માટે ૧૮૭૪ ઉમેદવારો ઉભા હતા. તમમજ આ ચૂંટણીમાં રાષ્ટ્રીય અને પ્રાદેશિક એવા ૭૭ રાજકીય પક્ષોએ ભાગ લીધો હતો. ચૂંટણી સમયે કોઇ વિશિષ્ટ મુદ્દ ન હતી.

લોકસભાની પ્રથમ સામાન્ય ચૂંટણીઓમાં ભારતીય રાષ્ટ્રીય કોંગ્રેસને ૩૬૪ બેઠકો ૪૪.૯ ટકા મતે મળી. જયારે સામે પક્ષે વિરોધ પક્ષના મતો વહંચાયેલા હતાં. સમાજવાદી પક્ષ અને કિસાન મજદૂર પક્ષે સંયુક્ત રીત ર૧ બેઠકો મેળવી જયારે સામ્યવાદી પક્ષોએ ૧૬ બેઠકો અને ભારતીય જનસંઘે 3 બેઠકો મેળવી. આમ, ચૂંટણીમાં લોકસભામાં ૩૭ અપક્ષ સભ્યો અને ૨ર સ્ત્રીઓ ચૂંટાઇ હતી. ત્યારબાદથી લઇને ૨૦૧૯ સુધી ભારતમાં ૧૭ લોકસભાની સામાન્ય ચૂંટણીઓ યોજાઇ ગઇ છે.

ભારતમાં ૧૬મી લોકસભાની સામાન્ય ચૂંટણી ૭ એપ્રિલ, ૨૦૧૪ થી ૧૨ મે, ૨૦૧૪ દરમિયાન કુલ નવ તબક્કામાં યોજાઇ હતી. આ ચૂંટણીમાં ભષ્ટાચાર, મોંધવારી, કુશાસન વગેરે મુખ્ચ મુદ્દા હતા. આ ચૂંટણીના પરિણામ ૧૬ મે, ૨૦૧૪ એ જાહેર કરવામાં આવ્યા હતા. તે મુજબ ૩૩૬ સીટો રાષ્ટ્રીય લોકતાંત્રિક ગઠબંધન (એન.ડી.એ.) અને ૨૮૨ બેઠક ભારતીય જનતા પાર્ટીન પ્રાપ્ત થઇ. જ્યારે સંયુક્ત પ્રગતિશીલ જોડાણને ૬૦ બેઠકો અને ભારતીય રાષ્ટ્રીય કોંગ્રેસને ૪૪ બેઠક પ્રાપ્ત થઇ હતી તથા અન્ય પક્ષોને ૧૪૭ બેઠકો પ્રાપ્ત થઇ હતી.

૨૦૧૪ની ૧૬મી લોકસભામાં બહમતીથી બનલલી રાષ્ટ્રીય લોકત્રાંતિક ગઠનબંધન (એન.ડી.એ.) સરકારે સ્થિરતા અને એકતા સાથે તેનું સત્ર પૂરં કય્યું હતું. તેથી એન.ડી.એ. ના નેતાઓન સંતોષ હતો. દેશમાં આ મોરચા સરકાર ની પ્રતિષ્ઠા પણ બંધાઇ હતી. તમમ આ સરકારે લોક કલ્યાણની યોજના શરૂ કરી તથા આર્થિક ક્ષેત્ર પણ પ્રગતિ કરી. તે ઉપરાંત ૨૦૧૪ થી ૨૦૧૯ સુંધી વિઘના દેશો સાથે તેમજ પડોશી દેશ સાથે પણ દ્વિપક્ષીય સંબંધો મજબૂત કર્યા તેથી આંતરરાષ્ટ્રીય ક્ષેત્ર પણ આ સરકારની પ્રતિષ્ઠા વધી.

ભારતમાં ૧૭ મી લોકસભાનું ગઠન કરવા માટે ૧૧ એપ્રિલથી ૧૯ મે ૨૦૧૯ સુધી સાત તબક્કામાં ૫૪ર બેઠકો માટે ચૂંટણી યોજાઇ હતી. આ ચૂંટણીના પરિણામ ૨૩ મે એ જાહેર કરવામાં આવ્યા હતાં. આ ચૂંટણીમાં ભાજપાના નેતૃત્વવાળા રાષ્ટ્રીય લોકશાહી મોરચાનો સ્પષ્ટ વિજય થયો. જેમાં રાષ્ટ્રીય લોકતાંત્રિક ગઠબંધન (એન.ડી.એ)ન ૩૫૩ બેઠકો મળી જેમાં ભાજપાને 303 બેઠકો પ્રાપ્ત થઇ જયારે સંયુક્ત પ્રગતિશીલ જોડાણને ૯૧ બેઠકો અને ભારતીય રાષ્ટ્રીય કોંગ્રેસને પર બેઠકો પ્રાપ્ત થઈ હતી. તથા અન્ય પક્ષોન ૯૮ બેઠકો પ્રાપ્ત થઇ હતી.

\section{વર્ષ-૨૦૧૯ની ચૂંટણી પૂર્વેની સ્થિતિ}

ભારતમાં ૧૬મી લોકસભાની વર્ષ ૨૦૧૪ની ચુંટણીમાં રાષ્ટ્રીય લોકતાંત્રિક ગઠબંધન (એન.ડી.એ.)ને ૩૩૬ બેઠકો પ્રાપ્ત થઈ હતી. જેમાંથી ભારતીય જનતા પાર્ટીન (ભાજપ) ૨૮ર બેઠકો મળી હતી. જ્યારે સંયુક્ત પ્રગતિશીલ જોડાણને (યુ.પી.એ) ૬૦ બેઠકો પ્રાપ્ત થઈ હતી જેમાંથી ભારતીય રાષ્ટ્રીય કોંગ્સ પક્ષને ૪૪ બેઠક પ્રાપ્ત થઈ હતી. તથા અન્ય પક્ષોને ૧૪૭ બેઠકો પ્રાપ્ત થઇ હતી 
લોકસભાની વર્ષ ૨૦૧૪ની ચૂંટણીમા રાષ્ટ્રીય લોકતાંત્રિક ગઠબંધન (એન.ડી.એ.)ને કુલ મતમાંથી ૩૮.૫\% મત પ્રાપ્ત થયા હતા જેમાંથી ૩૧.૫\% મત ભારતીય જનતા પાર્ટીન પ્રાપ્ત થયા હતા. જ્યારે સંયુક્ત પ્રગતિશીલ જોડાણને (યુ.પી.એ) ૨૩.૩\%મત મળ્યા હતા જેમાંથી ભારતીય રાષ્ટ્રીય કોંગ્રેસન ૧૯.૫\% મત મળ્યા હતા.

૨૦૧૪ની ૧૬મી લોકસભામાં બહુમતીથી બનેલી રાષ્ટ્રીય લોકતાંત્રિક ગઠબંધન (એન.ડી.એ.) સરકારે સ્થિરતા અને એકતા સાથે તેનું સત્ર પૂર્ણ કર્યું હતું. તેથી દેશમાં આ મોરચા સરકારની પ્રતિષ્ઠા પણ બંધાઈ હતી તમમજ આ સરકારે લોક કલ્યાણની યોજનાઓ શરૂ કરી તથા આર્થિક ક્ષેત્ર પણ પ્રગતિ કરી. તે ઉપરાંત ૨૦૧૯ની ચૂંટણી પૂર્વ પ્રચારમાં રાષ્ટ્રવાદ, ભષ્ટાચાર, નોટબંધી, સવર્ણોને અનામત તેમજ હિંદુત્વનો મુદ્દો એર સ્ટ્રાઇક, રામ મંદિર,તથા પાકિસ્તાન સાથેના વિવાદ વગેરે મુદ્દાઓ ચૂંટણી પૂર્વ પ્રચારમાં જોવા મળ્યા હતા.

\section{લોકસભાની વર્ષ-૨૦૧૯ની ચૂંટણીમાં મુખ્ય રાજકીય પક્ષોનો ચૂંટણી જાહેરનામુ}

\section{ભારતીય જનતા પાર્ટીનુ ચૂંટણી જાહેરનામુ:}

લોકસભાની વર્ષ-૨૦૧૯ની ચૂંટણીમાં ભારતીય જનતા પાર્ટીઓ આ મુદ્દાઓન લઈન ચૂંટણી ઝંપલાવ્યું હતુ.

* આતંકવાદ વિરુધ્ધ સુરક્ષા પૂરી પાડવામાં આવશ, રાષ્ટ્રીય સુરક્ષા વધુ મજબુત કરાશ, રક્ષા ક્ષેત્ર આત્મનિર્ભરતા પ્રાપ્ત કરાશે.

* ોલીસ બળોનું આધુનિકીકરણ

* નાગરિક્વ સુધારા અધિનિયમ

* ખેડૂત કલ્યાણ યોજનાઓ:

$>$ તમામ ખેરૂતો માટે "કિસાન સન્માન યોજના"

$>$ નાના અને સીમાંત ખૂડ્ત માટે પેન્શન

> ગ્રામીણ ક્ષેત્રોમાં ર૫ લાખ કરોડ રૂપિયાનું યોગદાન

$>$ व્યાજમુક્ત ખેડ્ત ક્રેડીટ કાર્ડ ઋણ

> "પ્રધાનમંત્રી ફસલ વીમા યોજનામાં" સ્વૈચ્છિક પંજીકરણ

> ખેતી દ્વારા ખેડૂત સશક્તિકરણ

* અર્થવ્યવસ્થા પાંચ ટ્રીલીયન ડોલરની બનાવવામાં આવશે.

> ૨૦૨૦ સુધી દરેક કુટ્ટુબન પાકું મકાન મળશે.

> જળજીવન મિશનની શરૂઆત થશે જે અંતર્ગત વર્ષ ૨૦૨૨ સુધી દરેક કુટુંબન પાઈપ-નળ દ્વારા પાણીની પૂર્તિ માટે "નળ થી જળ" અભિયાન ચલાવવામાં આવશે.

> ૨૦૨૨ સુધી દરેક ગ્રામ પંચાયત હાઈ-સ્પીડ ઓપ્ટીકલ ફાઈબરથી જોડાશે.

$>$ જળ ગ્રામ વિકાસને વધારવાના ઉદ્દેશથી શિક્ષા કેન્દ્રો, સ્વાસ્થ્ય કેન્દ્રો, તેમજ બજારોને ગામથી જોડવા માટે મોટા પાયા પર “ગ્રામીણ સડક ઉજાત" કાર્યક્રમ શરુ કરવામાં આવશે. 


\section{Parmar \& Dr. Hitesh Patel / Page 130-136}

> એક રાષ્ટ્ર એક ચૂંટણી (One Nation, One Election)

\section{ભારતીય રાષ્ટ્રીય કોંગેસ પક્ષનુ ચૂંટણી જાહેરનામુ}

લોકસભાની વર્ષ-૨૦૧૯ની ચૂંટણીમાં ભારતીય રાષ્ટ્રીય કોંગ્રેસે આ મુદ્દાઓન લઈન ચૂંટણી ઝંપલાવ્યું હતુ.

* રોજગાર : નવા સરકારી પદો નું સર્જન, વર્તમાન મંજૂર પદો પર સમયસર ભરતી

* ગરીબી :- વર્ષ ૨૦૩૦ સુધી ગરીબી નાબૂદ કરવા માટે કોંગ્રેસે ન્યુનમત આય યોજનાની જાહેરાત કરી હતી. જેમાં ભારત ની ૨૦\% સૌથી ગરીબ વસ્તીને ૭૨૦૦૦ રૂ. પ્રતિ વર્ષ આપવાની જાહેરાત કરી હતી.

* ઈન્ફાસ્ટ્રક્ચર રાષ્ટ્રીય રાજમાર્ગીનાં નિર્માણમાં ગતિ, તેના વિકાસ રાજમાર્ગો ના નિર્માણમાં રૂપરેખા, ગુણવત્તા, મેંઇન્ટેનન્સ અને કોન્ટ્રાકટરોને બાંધકામ પ્રત્ટે જવાબદારી નક્કી કરવામાં આવશે.

* અસંગઠિત ક્ષેત્ર ના મજૂરોન ન્યૂનતમ આવક પૂરી પાડવામાં આવશે.

* ખેડ્રનનું દેવું માફ કરવામાં આવશે.

* રાજકોષીય સ્થિરતા મેળવવી.

* રાજકોષીય ખાદ્ય ઓછી કરવી એ પ્રથમ લક્ષ્ય રહેશે.

• બેંકોની એન.પી.એ (NPA) નિયંત્રણ કરવામાં આવશે

* પૂર્વોત્તર રાજ્યોમાં વિશેષ દરજ્જા જાળવી રાખવામાં આવશ.

* પર્યાવરણ અને જળવાયુ પરિવર્તન માટે યોગ્ય પગલાં લેવાશે.

\section{૨૦૧૯ની ચૂંટણીનું આયોજન}

ભારતમાં ૧૭મી લોકસભાનું ગઠન કરવા માટે સામાન્ય ચૂંટણી ૭ તબકકામાં ૧૧ એપ્રિલથી ૧૯ મે સુધી આયોજિત કરવામાં આવી હતી. તેની વિગત નીચે મુજબ છે.

\begin{tabular}{|c|c|c|c|}
\hline તબક્કી & સમયગાળો & બેઠકો & રાજ્યો \\
\hline તબક્કો- ૧ & એપ્રિલ- ૧ ૧ & ૯૧ - બેઠકો & ૨૦ - રાજ્યો \\
\hline તબક્કો- ૨ & એપ્રિલ- ૧૮ & ૯૫ - બેઠકો & ૧૨ - રાજ્યો \\
\hline તબક્કી- 3 & એપ્રિલ- ૨૩ & ૧૧૭ - બેઠકો & ૧૫ - રાજ્યો \\
\hline તબક્કી- ૪ & એપ્રિલ- ૨૯ & ૭૧ - બેઠકો & O૮ - રાજ્યો \\
\hline તબક્કી- ૫ & મે- ૬ & ૫૦ - બેઠકો & ૦૭ - રાજ્યો \\
\hline તબક્કો- ૬ & મે- ૧૨ & પ૯ - બેઠકો & ૦૭ - રાજ્યો \\
\hline તબક્કી- 9 & મે- ૧૯ & પ૯ - બેઠકો & OC - રાજ્યો \\
\hline
\end{tabular}


૨૦૧૯ની ચૂંટણીનું પરિણામ

લોકસભા ચૂંટણી પરિણામ-૨૦૧૯

\begin{tabular}{|l|l|l|l|}
\hline ક્રમ & રાજકીય પક્ષ & બેઠક પર ઉભા રફેલ ઉમેદવારની સંખ્ચા & મળેલ બેઠક \\
\hline ૧ & ભારતીય જનતા પાર્ટી & ૪૩૬ & ૩૦૩ \\
\hline ૨ & ભારતીય રાષ્ટ્રીય કોંગ્રેસ & ૪૨૧ & ૫૨ \\
\hline 3 & દ્રવિડ મુનેત્ર કઝગમ & ૨૩ & ૨૩ \\
\hline ૪ & અખિલ ભારતીય તૃણમૂલ કોંગ્રેસ & ૬૨ & ૨૨ \\
\hline ૫ & યુવાજન શ્રમિક રાયથું કોંગ્રેસ પાર્ટી & ૨૫ & ૨૨ \\
\hline ૬ & શિવસેના & ૨૩ & ૧૮ \\
\hline ૭ & જનતાદળ (યુનાઇટેડ) & ૧૭ & ૧૬ \\
\hline$૮$ & બીજુ જનતાદળ & ૨૧ & ૧૨ \\
\hline$૯$ & બહુજન સમાજ પાર્ટી & ૩૮૩ & ૧૦ \\
\hline ૧૦ & અન્ય & & ૬૪ \\
\hline કુલ & & ૫૪૨ \\
\hline
\end{tabular}

લોકસભા ચૂંટણી- ૨૦૧૯માં ગઠબંધનને મળેલ બેઠકો

\begin{tabular}{|c|c|c|}
\hline ક્રમ & ગઠબંધનનું નામ & મળેલ સીટ \\
\hline$q$ & રાષ્ટ્રીય લોકતાંત્રિક ગઠબંધન (NDA) & 343 \\
\hline २ & સંયુક્ત પ્રગતિશીલ જોડાણ (UPA) & $\in q$ \\
\hline 3 & અન્ય & $\in C$ \\
\hline \multicolumn{2}{|c|}{ કુલ } & ૫૪ર \\
\hline
\end{tabular}

\section{૨૦૧૯ ચૂંટણી પરિણામોનું વિશ્લેષણ}

ભારતમાં ૧૭મી લોકસભાનું ગઠન કરવા માટે સામાન્ય ચૂંટણી દેશભરમાં ૭ તબક્કામાં ૧૧ એપ્રિલ થી ૧૯ મે ર૦૧૯ના સમયગાળા દરમિયાન $૭$ તબક્કામાં આયોજીત કરવામાં આવી હતી. જેનું પરિણામ ર૩ મે ૨૦૧૯ ના રોજ જાહેર કરવામાં આવ્યું હતું તે મુજબ રાષ્ટ્રીય લોકતાંત્રિક ગઠબંધન (એન.ડી.ઓ.)ને પ૪રમાંથી ૩પ૩ બેઠકો પર વિજય પ્રાપ્ત થઇ. જેમાંથી ભારતીય જનતા પાર્ટીન (ભાજપ) ૩૦૩ બેઠકો પ્રાપ્ત થઈ હતી. જ્યારે સંયુક્ત 
પ્રગતિશીલ જોડાણને (યુ.પી.એ) ૫૩રમાંથી ૯૧ બેઠકો મળી હતી જેમાંથી ભારતીય રાષ્ટ્રીય કોંગેસ પક્ષને ૪ર૧માંથી પર બેઠકો મળી. તથા અન્ય પક્ષોએ ૯૮ બેઠકો પર વિજય પ્રાપ્ત કરી હતી.

૨૦૧૯ની લોકસભાની ચૂંટણીમાં રાષ્ટ્રીય લોકતાંત્રિક ગઠબંધન (એન.ડી.એ.)ન ૩૫૩ બેઠકો પ્રાપ્ત થઈ હતી, જે વર્ષ ૨૦૧૪ની ચૂંટણીની સરખામણીમાં ૧૭ બેઠકોનો વધારો છે. જેમાંથી ભારતીય જનતા પાર્ટીન (ભાજપ) ૩०૩ બેઠકો પ્રાપ્ત થઈ હતી જે વર્ષ ૨૦૧૪ની ચૂંટણીની સરખામણીમાં ર૧ બેઠકોની વધારો છે. જ્યારે સામે પક્ષે સંયુક્ત પ્રગતિશીલ જોડાણન (યુ.પી.わ) ૨૦૧૯માં ૯૧ બેઠક પ્રાપ્ત થઈ હતી, જે વર્ષ ૨૦૧૪ની ચૂંટણીની સરખામણીમાં ૩૧ બેઠકોનો વધારો છે. જ્યારે ભારતીય રાષ્ટ્રીય કોંગેસ પક્ષન ૨૦૧૯માં પર બેઠકો પ્રાપ્ત થઈ હતી, જે વર્ષ ૨૦૧૪ની ચૂંટણીની સરખામણીમાં ૦૮ બેઠકોનો વધારો છે.

૨૦૧૯ની લોકસભાની ચૂંટણીમાં ભારતીય જનતા પાર્ટીન (ભાજપ) 3૭.૩૬\% મત મળ્યા હતા. જે ગત વર્ષની સરખામણીએ ૫.૮૬\% મતોનો વધ્ધારો છે. જ્યારે ભારતીય રાષ્ટ્રીય કોંગ્સેસન ૧૯.૪૭\% મત મળ્યા હતા જે ગતવર્ષ ની સરખામણીમાં લગભગ સમાન છે.

\section{ઉપસંહાર}

લોકસભાની વર્ષ ૨૦૧૯ની ચૂંટણીના આવેલ પરિણામોના આધારે કહી શકાય કે ભારતના લોકો ફક્ત જ્ઞાતિ જોઇને મતદાન કરતા નથી. તેઓએ સક્ષમ નેતૃત્વ, પ્રજાના પ્રશ્જીન વાંચા આપે, તેમને વોટ આપ્યો છે. લોકસભાની વર્ષ ૨૦૧૯ની ચૂંટણીના પરિણામો એ એવા સંકેત આપ્યા છે કે જ્ઞાતિ આધારિત રાજકારણનો સમય હવે ધીરે ધીરે સમાપ્ત થઈ રહ્યો છે. કોઈપણ જ્ઞાતિ ચોક્કસ પાર્ટીની વોટબેંક રહી નથી. આવનાર ચૂંટણીઓના મુખ્ય મુદ્દાઓ રોજગાર, શિક્ષણ, ભષ્ટાયાર, ગરીબી નાબૂદી, આરોગ્ય વગેરે જેવા હશે. અને ભારતના લોકો હવે સરકારના કાર્યોનું મૂલ્યાંકન કરીને અગામી ચૂંટણીમાં મતદાન કરતા જોવા મળશે. 
१. પुસ્તકો:

I. India's 2019 Elections:- Edited by- Paul Wallace

II. Modi Mandate-2019 - Pradeep Bhandari

III. 2019- How Modi Won India:- Rajdeep Sardesai

IV. ભારતીય રાજકારણ - પોપ્યુલર પ્રકાશન -ડો. ગજેન્દ્ર બી. શુક્લ

૨. સમાચારપત્ર :

દિવ્ય ભાસ્કર, સંદેશ, નવગુજરાત સમય, ધ હિન્દુ, ઇન્ડિયન એક્ષપ્રેસ

3. વેબસાઈટ:

General election 2019- Election Commission of India

૪. યુટ્યુબ ચેનલ:

I. Rajysabha TV

II. DD News

III. Study IQ

૫. ભારતીય જનતા પક્ષ મેનીફેक्ष्टर -૨૦૧૯

૬. ભારતીય રાષ્ટ્રીય કોંગેસ પક્ષ મેનીફેक્ષ્ટ -૨૦૧૯

\author{
Mr. Parmar Sunil G. \\ Research Scholar (Ph.D.) Reg. No. 8839 \\ Dept. of Political Science \\ Gujarat University, Ahmedabad \\ \& \\ Dr. Hitesh R. Patel \\ (Guide) \\ Assi. Prof. Dept. of Political Science \\ Gujarat University, Ahmedabad
}

